\title{
Dimensión vertical oclusal mediante antropometría de los dedos de la mano. Validación del método antropométrico de Ladda
}

\section{Occlusal vertical dimension by anthropometry of fingers Validation of Ladda's anthropometric method}

\author{
Enrique Fernández ${ }^{\star}$, Patricio Jaramillo ${ }^{5}$, Héctor González ${ }^{2}$, Jorge Nakouzi ${ }^{3}$, Tatiana Padilla ${ }^{4}$
}

1. Especialista en Rehabilitación Oral, CONACEO, Posgrado de Especialización en Rehabilitación Oral, Universidad San Sebastián y Universidad Nacional Andrés Bello, Santiago, Chile. 2. Especialista en Rehabilitación Oral, CONACEO, Posgrado de Especialización en Rehabilitación Oral, Universidad Nacional Andrés Bello, Santiago, Chile.

3. Facultad de Odontología, Universidad Nacional Andrés Bello, Santiago, Chile. Especialista en Rehabilitación Oral, Universidad Nacional Andrés Bello.

4. Odontóloga, Universidad de Cuenca, Ecuador. Especialista en Rehabilitación Oral, Universidad Nacional Andrés Bello.

5. Cirujano Dentista, Universidad Nacional Andrés Bello, Santiago, Chile.

* Correspondencia autor. Prof.Dr. Enrique Fernández M. | Facultad de Odontología, Universidad San Sebastián. Av.Bellavista \#7, CP 8420524, Recoleta, CHILE. | e-mail: enrique. fernandez@uss.cl

Trabajo recibido el 21/05/2017. Aprobado para su publicación el 20/08/2017

\section{RESUMEN}

Objetivo: Validar en población chilena, el método antropométrico indio de Ladda que estableció en la población chilena una correlación positiva entre la dimensión vertical oclusal y longitud de ciertos dedos de la mano. Materiales y métodos: Estudio de tipo transversal, 151 adultos jóvenes, 76 hombres y 75 mujeres. Se replicó el trabajo referencial, utilizando un pie de metro digital, realizando mediciones de los dedos índice y meñique y la distancia desde la punta del pulgar a la punta del índice de la mano derecha, correlacionando las mediciones obtenidas con la dimensión vertical oclusal y aplicando el coeficiente de correlación de Pearson. Resultados: La dimensión vertical oclusal presenta correlación estadísticamente significativa con los parámetros estudiados. En hombres, la correlación fue más consistente con la longitud del dedo índice ( $r=0.908)$, mientras que, en las mujeres, fue más consistente con la longitud del dedo meñique $(r=0.827)$. Conclusiones: Las variaciones obtenidas caen dentro del intervalo de $3-4 \mathrm{~mm}$, estando en consonancia con otros métodos antropométricos publicados, confirmando la predictibilidad de la dimensión vertical oclusal obtenida mediante este método objetivo. El procedimiento es sencillo, económico y no invasivo, por lo que podría ser recomendado para la práctica clínica cotidiana.

PALABRAS CLAVE

Dedos, Pulgar, Dimensión vertical oclusal

Rev. Clin. Periodoncia Implantol. Rehabil. Oral Vol. 10(3); 149-152, 2017.

\section{ABSTRACT}

Objective: The purpose of this study was to validate in the Chilean population, the anthropometric method by Ladda which found a significant correlation between the length of the fingers and the vertical dimension of occlusion. Materials and methods: A cross-sectional study, with 151 Chileans: 76 males and 75 females. Anthropometric measurements of vertical dimension of occlusion, length of index finger, length of little finger, and distance from tip of thumb to tip of the index finger of the right hand were recorded clinically using digital Vernier caliper. Correlation was studied using Pearson's coefficient. Results: Vertical dimension of occlusion was significantly correlated with all the parameters analyzed. In males, correlation of vertical dimension of occlusion was stronger with the length of the index finger $(r=0.908)$ whereas in females, it was stronger with the length of the little finger $(r=0.827)$. Conclusions: Since the variations between vertical dimension of occlusion and finger lengths are within the range of 3-4 $\mathrm{mm}$, as with other anthropometric methods published, VDO prediction through this method is reliable, and reproducible. This method is simple, economic, and non-invasive; hence, it could be recommended for everyday clinical practice.

KEYWORDS

Fingers, Thumb, Vertical dimension of occlusion.

Rev. Clin. Periodoncia Implantol. Rehabil. Oral Vol. 10(3); 149-152, 2017. 


\section{INTRODUCCIÓN}

La dimensión vertical (DV) se define de acuerdo al glosario de términos prostodónticos, como la distancia entre dos puntos anatómicos determinados, generalmente ubicados en la base de la nariz y en el mentón, coincidentes con la línea media.(1) Se conoce como dimensión vertical oclusal (DVO) la dimensión vertical registrada cuando los dientes se encuentran ocluyendo entre sí; su determinación es una etapa crítica en el éxito rehabilitador del desdentado total o parcial, especialmente cuando no se cuenta con referencias de soporte oclusal. Puede encontrarse "alterada" debido a un sinnúmero de causas y el procedimiento para determinarla de manera correcta y fácilmente, se ha tornado un tema controversial. Una DVO imprecisa afecta tanto estética como función, pues podría generar problemas en la fono articulación, daños en las estructuras periodontales, mialgias, cefaleas, entre otros..$^{(2-3)}$

Establecida la relevancia de este parámetro, se han planteado a lo largo del tiempo varios métodos para su determinación: subjetivos y objetivos y de naturaleza cefalométrica, craneométrica y antropométrica. ${ }^{(4-5)}$

Se debe tomar en cuenta que los métodos diseñados para la medición de la DVO a menudo incorporan un rango importante de subjetividad por parte del operador. A pesar de todos los esfuerzos, varios métodos que se usan en la actualidad requieren una mayor complejidad, tiempo, instrumentos y equipos especiales, y no se obtienen siempre los mismos resultados, por lo que no existe un consenso sobre que método sería el más confiable, repetible y de uso ideal para poder determinar la DVO exacta. Por ello se continúa recomendando el uso de más de un método para cada caso. ${ }^{(6)}$

Leonardo da Vinci y $\mathrm{McGee}^{(7)}$ relacionaron varias medidas antropométricas a la DVO, como por ejemplo su similitud con la distancia del canto externo de un ojo al canto interno del otro, altura de la oreja, el doble del largo del ojo, distancia interpupilar y la medida del largo de la nariz en su línea media. Además, desde la antigüedad se ha usado medidas antropométricas para determinar proporciones de partes del cuerpo siguiendo lo que se conoce como las proporciones áuricas. ${ }^{\left({ }^{8}\right.}$ Dentro de esta misma línea, por mucho tiempo se ha considerado que la DVO es proporcional a la medida de la distancia desde la punta del pulgar a la punta del índice cuando se realiza una ligera presión para juntarlos. ${ }^{(9)}$

Ladda et al. ${ }^{(10)}$ evaluaron la correlación entre la DVO y el largo del dedo índice, largo del dedo meñique y distancia de la punta del pulgar a la punta del dedo índice, encontrando que la mayor correlación con la DVO fue con la longitud del dedo índice en hombres y del dedo meñique en mujeres.

El objetivo de nuestra investigación fue validar en población chilena los resultados del estudio de Ladda et al, de modo tal que podamos contar con otro procedimiento clínico objetivo, que se puede aplicar por el profesional en la gran mayoría de los pacientes y así en conjunto con otros métodos, obtener un registro de DVO más certero.

\section{MATERIALES Y MÉTODOS}

Para este estudio, se seleccionaron 151 individuos divididos en 76 hombres y 75 mujeres, entre los alumnos de pregrado de la Facultad de Odontología de la Universidad Andrés Bello, sede Santiago, con un rango etario de 18 y 28 años

Todos ellos cumplían con los siguientes criterios de inclusión: ser chilenos, tener su dentición completa (con o sin presencia de terceros molares) y presentar una máxima intercuspidación estable.

Se excluyeron de este estudio, sujetos con anomalías dentomaxilares en sentido vertical (mordida abierta o mordida profunda), individuos con facetas de desgaste mayores a grado II, que hubiesen sido sometidos a tratamiento ortodóncico, quirúrgico, o presentasen tratamientos restauradores o rehabilitadores extensos, en cuyo procedimiento se haya modificado la dimensión vertical oclusal. Finalmente se excluyeron también, aquellos que tuviesen alguna anomalía de forma o tamaño en los dedos de la mano derecha.

Sólo se establecieron registros de los dedos de la mano derecha para evitar sesgos. Conjuntamente se obtuvo el consentimiento informado de todos los voluntarios que participaron del estudio. Dichos documentos obran en poder de los autores.

A todos los individuos seleccionados, se les realizó las siguientes 4 mediciones, por un sólo operador, previamente calibrado y entrenado, utilizando un pie de metro digital, remedando lo llevado a cabo por Ladda et al.

1. Longitud del dedo índice: Con la mano recta y plana, en supinación, medimos desde la punta del dedo hasta el último pliegue palmar. Fig.1a

2. Longitud del dedo meñique: Con la mano recta y plana, en supinación, medimos desde la punta del dedo hasta el último pliegue palmar, como se observa en la Fig. 1b

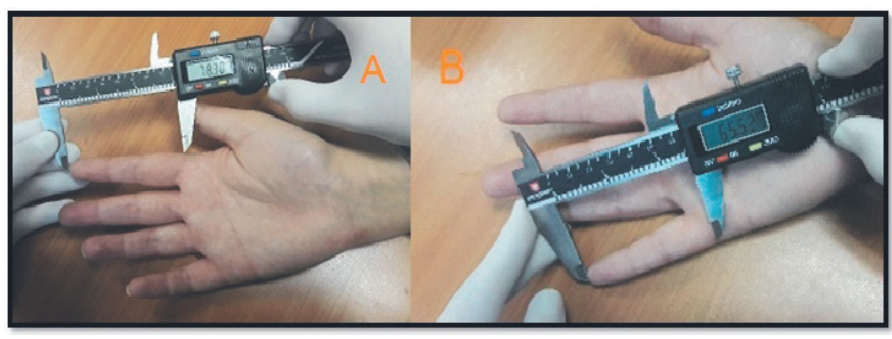

Figura 1.

3. Dimensión Vertical Oclusal: Se solicitó a los pacientes ocluir en una relación de máxima intercuspidación (MIC), manteniendo sus labios en reposo, con la cabeza bien estabilizada. El extremo inferior del pie de metro se colocó firmemente por debajo del mentón mientras que el extremo superior se elevó hasta que tocara ligeramente la base del tabique nasal. Fig.2

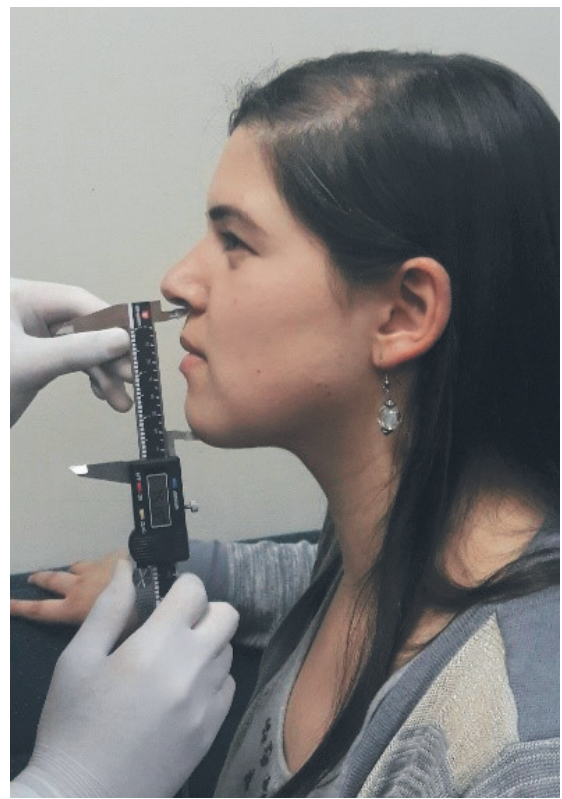

Figura 2.

Para todos los parámetros del estudio se calculó el promedio, desviación estándar, mediana y rango intercuartil. La correlación fue estudiada utilizando el Coeficiente de Correlación de Pearson. Se ejecutó una regresión lineal y se prepararon ecuaciones para estimar la DVO mediante el programa STATA ${ }^{\circledR}$ versión 14.2, Texas, USA.

\section{RESULTADOS}

Los análisis descriptivos de las variables estudiadas se muestran en la tabla 1.

Se observa que en hombres el valor promedio de la DVO fue de $74,53 \mathrm{~mm}$, en un rango entre $66,56 \mathrm{~mm}$ y $81,84 \mathrm{~mm}$, mientras que en mujeres el valor promedio de la DVO fue de $61,77 \mathrm{~mm}$ en un rango entre 53,94 y $70,11 \mathrm{~mm}$. Por consiguiente, la DVO fue mayor en hombres que en mujeres.

Para las longitudes de los dedos, en hombres la longitud del dedo índice tuvo un promedio de $74,62 \mathrm{~mm}$, mientras que en mujeres fue de $69,34 \mathrm{~mm}$. La longitud promedio del dedo meñique en hombres fue de $64,33 \mathrm{~mm}$ y en mujeres el promedio fue de $59,57 \mathrm{~mm}$. Por último, la media para la distancia entre la punta del dedo índice y la punta del dedo pulgar fue de $60,48 \mathrm{~mm}$ en hombres y $55,61 \mathrm{~mm}$ en mujeres.

Para estimar la correlación entre las variables estudiadas y la DVO, se utilizó el coeficiente de correlación de Pearson ( $r$ ), cuyos resultados más relevantes se muestran en la tabla 2 .

Todos los parámetros estudiados se correlacionaron positiva significativamente con la DVO, siendo la mayor correlación, la existente entre la longitud del dedo índice y la DVO ( $r=0.808)$, mientras que con la variable "distancia entre la punta del dedo índice y punta del dedo pulgar" la correlación es sólo regular ( $r=0.569)$.

Tanto en hombres como en mujeres, la DVO se relaciona de manera 
Tabla 1: Análisis descriptivo de las variables, DVO, longitud de dedos y género.

\begin{tabular}{|c|c|c|c|c|c|c|c|}
\hline & & Mínimo & Máximo & Media & $\mathrm{DE}^{*}$ & Mediana & IRQ* \\
\hline \multirow{3}{*}{$\begin{array}{l}\text { DVO } \\
(\mathrm{mm})\end{array}$} & $\delta \mathrm{H}$ & 66.56 & 81.84 & 74.53224 & 3.310112 & 74.585 & 4.005001 \\
\hline & ${ }_{M}$ & 53.94 & 70.11 & 61.77693 & 3.518308 & 62.35 & 5.490002 \\
\hline & Total & 53.94 & 81.84 & 68.19682 & 7.247686 & 68.25 & 12.24 \\
\hline \multirow{3}{*}{$\begin{array}{l}\text { Índice } \\
\text { (mm) }\end{array}$} & ${ }^{2} \mathrm{H}$ & 65.61 & 81.05 & 74.61987 & 3.475096 & 74.95 & 4.350002 \\
\hline & qM & 59.61 & 81.05 & 69.34467 & 4.17262 & 68.68 & 5.580002 \\
\hline & Total & 59.61 & 81.05 & 71.99974 & 4.650856 & 72.69 & 7.029999 \\
\hline \multirow{3}{*}{$\begin{array}{l}\text { Meñique } \\
(\mathrm{mm})\end{array}$} & ${ }^{2} \mathrm{H}$ & 54.36 & 71.48 & 64.33 & 3.715855 & 64.365 & 5.124998 \\
\hline & $+\mathrm{M}$ & 52.08 & 71.68 & 59.5732 & 3.914682 & 59.09 & 5.380001 \\
\hline & Total & 52.08 & 71.68 & 61.96735 & 4.489793 & 62 & 6.73 \\
\hline \multirow{3}{*}{$\begin{array}{l}\text { Índice - } \\
\text { Pulgar } \\
\text { (mm) }\end{array}$} & $\widehat{J} \mathrm{H}$ & 51.59 & 70.93 & 60.47816 & 4.338952 & 60.165 & 5.634998 \\
\hline & $+\mathrm{M}$ & 44.68 & 65.5 & 55.60587 & 4.664924 & 55.32 & 7.399998 \\
\hline & Total & 44.68 & 70.93 & 58.05815 & 5.111071 & 58.62 & 7.360001 \\
\hline
\end{tabular}

$D E=$ Desviación Estándar, $I R Q=$ rango intercuartil

Tabla 2: Correlación entre la DVO y longitud del dedo índice, meñique, según género, de acuerdo a coeficiente de correlación de Pearson.

\begin{tabular}{cccc}
\hline Género & $\begin{array}{c}\text { Coeficiente de } \\
\text { Correlación }\end{array}$ & Índice & Meñique \\
\hline DVO en & $\mathrm{R}$ & 0.9080 & 0.6101 \\
Hombres & $\mathrm{N}$ & 76 & 76 \\
\hline DVO en & $\mathrm{R}$ & 0.7008 & 0.8270 \\
Mujeres & $\mathrm{N}$ & 75 & 75 \\
\hline
\end{tabular}

$r=$ Coeficiente de correlación de Pearson (Calificación de la correlación: 0.65 a 1 - Buena; 0.4 a 0.649 - Regular; 0 a $<0.4$ - Mala).

positiva y significativa con la longitud de los dedos índice y meñique, respectivamente. En hombres se observa una mayor correlación con el parámetro "longitud del dedo índice" ( $r=0.908)$, mientras que en mujeres la correlación más fuerte es con la longitud del dedo meñique $(r=0.827)$.

Por otra parte, la correlación existente entre la DVO y la distancia entre la punta del dedo índice y la punta del dedo pulgar fue calculada mediante el coeficiente de correlación de Spearman, por no presentarse linealidad en los gráficos. Además, esta relación fue la más débil $(r=0.2438$ en hombres y $r=0.4019$ en mujeres $)$.

Se realizó una regresión lineal de los datos, utilizando todos los parámetros estudiados para predecir la DVO. Los resultados principales de estas regresiones se muestran en las Figs. 3 y 4

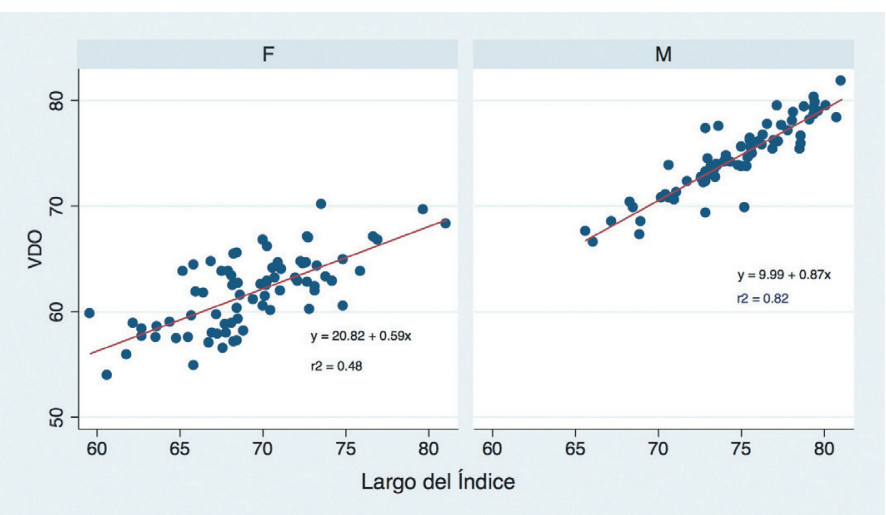

Figure 3.

En la tabla 3, apreciamos las ecuaciones de regresión que complementan el proceso para obtener la DVO, tanto en hombres como en mujeres.

La determinación de la DVO utilizando las ecuaciones de regresión para la longitud del dedo índice, tuvo un error estándar de $\pm 3,466 \mathrm{~mm}$ y $\pm 4,891 \mathrm{~mm}$, en hombres y mujeres respectivamente. Por su parte, la determinación de la DVO usando las ecuaciones de regresión para la longitud del dedo meñique, tuvo un error estándar de $\pm 5,287 \mathrm{~mm}$ en hombres y de $\pm 3,530 \mathrm{~mm}$ en mujeres. Finalmente, la determinación de la DVO usando las ecuaciones de regresión para la distancia entre la punta del dedo índice a la punta del dedo pulgar, tuvo un error estándar en hombres de $\pm 5,157 \mathrm{~mm}$ y en mujeres de $\pm 4,472 \mathrm{~mm}$.

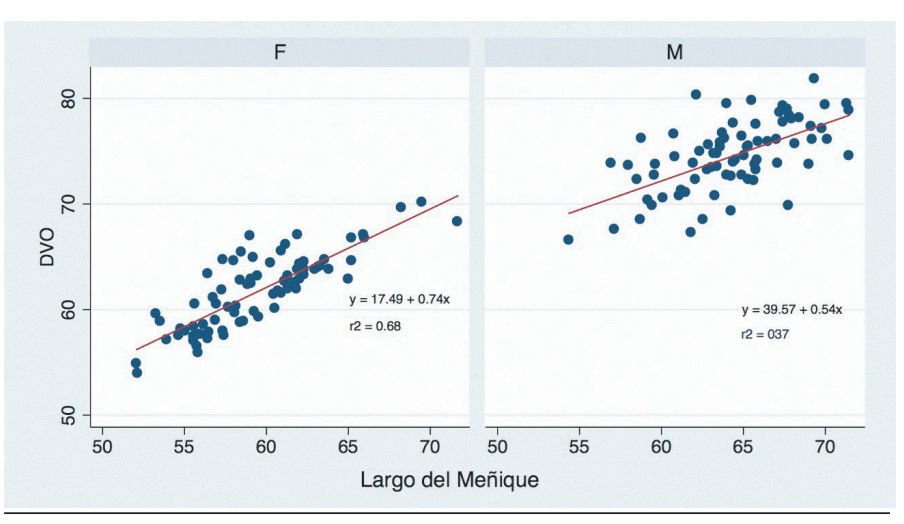

Figure 4.

Tabla 3: Resumen de regresiones lineales de las variables, según género.

\begin{tabular}{|c|c|c|c|c|c|}
\hline Género & $\begin{array}{c}\text { Variable } \\
\text { dependiente }\end{array}$ & $\begin{array}{c}\text { Variable } \\
\text { Independiente }\end{array}$ & $\begin{array}{l}\text { Ecuación de } \\
\text { regresión }\end{array}$ & $\begin{array}{c}\text { R2 (\%) } \\
\text { Ajustado }\end{array}$ & SE \\
\hline \multirow{3}{*}{ Masculino } & \multirow{3}{*}{ DVO (Y) } & A & $\begin{array}{c}Y=9.99+ \\
0.87 \mathrm{~A}\end{array}$ & 82.20 & $\begin{array}{c} \pm \\
3.466\end{array}$ \\
\hline & & B & $\begin{array}{c}Y=39.57+ \\
0.54 \mathrm{~B}\end{array}$ & 36.37 & $\stackrel{ \pm}{5.287}$ \\
\hline & & C & $\begin{array}{c}Y=61.46+ \\
0.22 \mathrm{C}\end{array}$ & 6.78 & $\begin{array}{c} \pm \\
5.157\end{array}$ \\
\hline \multirow{3}{*}{ Femenino } & \multirow{3}{*}{ DVO (Y) } & A & $\begin{array}{c}Y=20.82+ \\
0.59 \mathrm{~A}\end{array}$ & 48.41 & $\stackrel{ \pm}{ \pm}$ \\
\hline & & B & $\begin{array}{c}Y=17.49+ \\
0.74 \mathrm{~B}\end{array}$ & 67.96 & $\begin{array}{c} \pm \\
3.530\end{array}$ \\
\hline & & C & $\begin{array}{c}Y=44.21+ \\
0.32 \mathrm{C}\end{array}$ & 16.42 & $\stackrel{ \pm}{ \pm .472}$ \\
\hline
\end{tabular}

( $Y=D V O, A=$ longitud del dedo índice, $B=$ longitud del dedo meñique, $C=$ distancia entre la punta del dedo índice y la punta del dedo pulgar, R2 = coeficiente de determinación ajustado, SE = Error Estándar).

\section{DISCUSIÓN}

Establecer la DVO es una fase de la determinación de las relaciones cráneo mandibulares en la que se presentan mayores dificultades. La altura del tercio inferior del rostro es variable de acuerdo a las diferentes posiciones que pueda adoptar la mandíbula en el plano vertical en concordancia con las distintas funciones que se realicen. ${ }^{(11)}$ Cuando una DVO es alterada de manera considerable se pueden presentar problemas en la fonación, masticación e incluso problemas articulares temporomandibulares. ${ }^{(12)}$

Determinar la DVO no es un proceso exacto y muchos profesionales llegan a la misma, utilizando varios métodos, subjetivos y objetivos. ${ }^{(9,13}$, 14,15

Algunos autores ponen en duda la precisión de métodos subjetivos como el de la deglución ${ }^{(13)}$, el fonético ${ }^{(14)}$, análisis estético ${ }^{(9)}$, posición 
postural mandibular(15), y por otro lado, métodos objetivos como el de Knebelmann, Willis o bien cefalométricos, que si bien son más precisos, resultan más complejos de aplicar y se requiere de instrumentos específicos de más difícil acceso. Por ello y por la variación de valores en personas de distinta etnia o género, se ha sugerido el uso de más de un método para garantizar una correcta DVO. ${ }^{(16)}$

En búsqueda de un método de mayor sencillez, precisión y confiabilidad, se evaluó la relación antropométrica entre la longitud de los dedos índice y meñique de la mano, así como la distancia entre la punta del pulgar a la punta del índice y la eventual concordancia con la DVO, replicando gran parte de la metodología aplicada en el estudio indio.

Nuestros resultados apuntan en la misma dirección ratificando en población chilena joven, la existencia de una correlación significativa entre la DVO y la longitud de algunos de los dedos de la mano.

Los valores medios de las variables antropométricas verificadas en el presente estudio, están en concordancia con las obtenidas por otros autores de otras latitudes ${ }^{(17,18,19)}$, especialmente para las longitudes de los dedos índice y meñique, para los cuales se obtuvieron los menores valores de error estándar calculando la DVO $(3,47 \mathrm{~mm}$ para el índice en hombres y $3,53 \mathrm{~mm}$ para el meñique en mujeres).

No fue posible encontrar ningún estudio en el que el parámetro "distancia entre la punta del dedo índice a la punta del dedo pulgar" estuviese considerado, salvo el referencial de Ladda et al ${ }^{(10)}$, con el cual los valores medios de dicha longitud también están en concordancia. Sumado a esto, mediante la aplicación de un modelo matemático, la DVO puede estimarse a partir de esta distancia utilizando una ecuación de regresión, la que, sin embargo, presenta los mayores valores de error estándar y los menores valores de $r^{2}$ ajustado, por lo que nos parece pertinente descartar este parámetro.

Los resultados indican que mediciones antropométricas de la longitud de los dedos de la mano pueden servir como guía básica en la estimación de la DVO, ofreciendo ventajas significativas en el éxito del tratamiento protésico. Al tratarse de mediciones objetivas, en lugar de criterios subjetivos, la diferencia de percepción se elimina o bien se reduce a su mínima expresión.

\section{CONCLUSIONES}

Al igual que en población india, nuestro estudio determinó que existe una correlación positiva y significativa entre dos de las tres variables antropométricas estudiadas y la DVO.

La correlación encontrada para la distancia medida, entre la punta del dedo índice y la punta del dedo pulgar, es solo regular comparada con las correlaciones de las medidas anteriormente descritas.
El parámetro más certero para determinar la DVO en hombres es la longitud del dedo índice, con un error estándar de $\pm 3.466 \mathrm{~mm}$., mientras que en mujeres es la longitud del dedo meñique, con un error estándar de $\pm 3.530 \mathrm{~mm}$. Es así que, la DVO estimada utilizando este método se situaría en un rango entre $3-4 \mathrm{~mm}$, lo que es significativamente menor comparado con otros métodos, en los que se verifica un rango entre $0-14 \mathrm{~mm} \cdot{ }^{(20,21)}$

Además de constituirse en un método práctico, súmase una aplicación simple, económica, no invasiva, rápida, con buen grado de fiabilidad y reproducibilidad, que no requiere de radiografías o dispositivos de medición sofisticados ni de gran experiencia para realizarla correctamente.

Sin embargo, el operador debe tener en cuenta que la DVO es el resultado de un equilibrio músculo-esquelético. Por lo tanto, debería adoptarse en todas las etapas de rehabilitación más de un método para determinar o devolver una correcta DVO, con el fin de maximizar los beneficios y minimizar los daños en el sistema estomatognático.

Las limitaciones de este estudio fueron principalmente que los individuos no se categorizaron según biotipos faciales, ni según tipo de oclusión presente. Para aumentar el caudal de evidencias empíricas que sustentan estos hallazgos, es deseable llevar a cabo más estudios siguiendo una línea de investigación clínica que incluya análisis similares en otras poblaciones e incorporando otras variables - aporte imagenológico - en la perspectiva de obtener resultados que provean de técnicas o métodos que puedan ser aceptados y aplicados clínicamente de manera universal.

\section{RELEVANCIA CLÍNICA}

La dimensión vertical oclusal es una parte muy relevante dentro de una correcta Rehabilitación oral. No existe un consenso de cuál es el método ideal para obtenerla. Con el estudio que realizamos, pretendimos validar en la población chilena, el método antropométrico de Ladda. Obtuvimos como resultado una correlación positiva entre medidas antropométricas de los dedos índice y meñique de la mano derecha con la dimensión vertical oclusal, sugiriendo el uso de este método como confiable, sencillo y reproducible para la práctica odontológica diaria.

\section{CONFLICTO DE INTERÉS}

Los autores declaran no tener ningún conflicto de interés.

\section{FUENTE DE FINANCIAMIENTO}

En cuanto a las fuentes de apoyo financiero no existieron tales.

\section{Bibliografía}

1. The Glossary of Prosthodontic Terms. J Prosthet Dent. 2005;94:10-92. 2. Turner KA, Missirlian DM. Restoration of the extremely worn dentition. J Prosthet Dent. 1984: 52:467-474

3. Mohindra NK, Bulman JS. The effect of increasing vertical dimension of occlusion on facial aesthetics. Br Dent J. 2002; 192:164-168.

4. Turrell A. Clinical assessment of vertical dimension. J. Prosthet Dent. 1972(28) 3: $238-46$

5. Quiroga R., Sierra M, del Pozo J., Quiroga R. Dimensión vertical oclusal comparación de 2 métodos cefalométricos. Rev Clin Periodoncia Implantol Rehabi Oral. 2016;9(3):264-270

6. Rivera-Morales WC, Mohl ND. Relationship of occlusal vertical dimension to the health of the masticatory system. J Prosthet Dent. 1991; 65:547-553.

7. McGee GF. Use of facial measurements in determining vertical dimension. J Am Dent Assoc. 1947; 35:342-50

8. Willis FM. Features of the face involved in full denture prosthesis. Den Cosmos1935; 77:851-854

9. Misch CE. Clinical indications for altering vertical dimension of occlusion. Objective vs subjective methods for determining vertical dimension of occlusion. Quintessece Int. 2000;31:280-282.

10. Ladda R, Kasat VO, Bhandari AJ. A new technique to determine vertica dimension of occlusion from anthropometric measurements of fingers. Indian J Dent Res. 2013;24:316-320.

11. Winkler S. "Prostodoncia Total". México: Interamericana;1982.

12. Kawabe, S. "Kawabe's Dentaduras Totales". Primera edición. Colombia: Actualidades Médico Odontológicas Latinoamericana, C.A; 1993.
13. Boucher, C. Prótesis para el desdentado total. Buenos Aires- Argentina: Ed. Mundi; 1977. 216-290.

14. Dawson P. Phonetic method of measuring occlusal vertical dimension. Bay View Dental Laboratory. Disponible en: http://www.bvdl.com/wp-content/uploads/2011/06/ Phonetic-Method-of-Measuring-Occlusal-Vertical-Dimension.pdf

15. Romo F., Jorquera C, Irribarra R. Determinación de la dimensión vertical oclusal a través de la distancia clínica ángulo externo del ojo al surco tragus facial. Rev Dent Chile. 2009;100(3)26-33.

16. Banasr FH, Al-Rafah EM. Evaluation of divine proportion ratio as a method for registration of rest vertical dimension using statistical analysis in completely edentulous patients. Smile Dent J. 2012;7(1)44-9.

17. Danborno B, Adebisi S, Adelaiye A, Ojo S. Estimation of height and weight from the lengths of second and fourth digits in Nigerians. Internet J Forensic Sci. 2009;3:2.

18. Kanchan T, Kumar GP, Menezes RG, Rastogi P, Rao PP, Menon A, et al. Sexual dimorphism of the index to ring finger ratio in South Indian adolescents. J Forensic Leg Med. 2010;17:243-6.

19. Nag A, Nag P, Desai H. Hand anthropometry of Indian women. Indian J Med Res. 2003;117:260-9.

20. Silverman MM, Accurate measurement of vertical dimension by phonetics and the speaking centric speace, Part I. Dent Dig. 1951;57:261-5.

21. Benediktsson $E$. Variation in tongue and jaw position in "S" sound production in Relation to Front Teeth Occlusion. Acta Odontol Scand. 1958:15:275-303. 